\title{
BMJ A Protocol for the Pharmacokinetics of OPen Enteric Coated Mycophenolate Sodium in Lupus Nephritis (POEMSLUN): an open-label, randomised controlled trial
}

To cite: Ranganathan $D$, John GT, Healy $\mathrm{H}$, et al. A Protocol for the

Pharmacokinetics of Enteric Coated Mycophenolate Sodium in Lupus Nephritis (POEMSLUN): an open-label, randomised controlled trial. BMJ Open 2013;3:e003511. doi:10.1136/bmjopen-2013003511

- Prepublication history for this paper is available online. To view these files please visit the journal online (http://dx.doi.org/10.1136/ bmjopen-2013-003511).

Received 1 July 2013 Revised 8 July 2013 Accepted 10 July 2013
For numbered affiliations see end of article.

\section{Correspondence to} Dr Dwarakanathan Ranganathan; dwarakanathan ranganathan@health.qld. gov.au

\section{ABSTRACT}

Introduction: Mycophenolate sodium, an entericcoated tablet (EC-MPS), is as effective and safe as mycophenolate mofetil (MMF) in preventing transplant rejection. EC-MPS and MMF improve the outcome of severe lupus nephritis (LN) and have fewer side effects than pulsed intravenous cyclophosphamide. Blood concentrations of mycophenolic acid (MPA), the active metabolite of EC-MPS, vary between participants despite fixed dosing. Interpatient variability has been studied in transplantation, but not well documented in LN. The relationship between MPA concentration and its clinical effect on LN has not been described.

Methods and analysis: This is a prospective, openlabel, randomised controlled trial. -32 participants with LN who meet the inclusion and exclusion criteria will be randomised into two groups: one receiving a fixed dose of EC-MPS and the second, a dosing regimen that is titrated with therapeutic drug monitoring. Included participants will have blood sampled over a period of 8-12 $\mathrm{h}$ on three different occasions. Pharmacokinetic parameters will be calculated using non-compartmental methods.

Ethics and dissemination: The Human Research and Ethics Committee of the Royal Brisbane Women's Hospital have approved this study. The study is registered with Australian and New Zealand Clinical Trials Registry-ACTRN12611000798965 We planned to present the de-identified information at conferences and publish the results in medical journals.

Trial Registration: ACTRN12611000798965

\section{BACKGROUND}

Mycophenolate mofetil (MMF), the first prodrug of mycophenolic acid (MPA), has been used in kidney transplant recipients for the prevention of acute rejection in combination with steroids and calcineurin inhibitors

\section{ARTICLE SUMMARY}

Article focus

- Interpatient variability of blood concentrations of mycophenolic acid (MPA), the active metabolite of enteric-coated mycophenolate sodium (EC-MPS), has been studied in transplantation, but not well documented in lupus nephritis (LN).

- This article describes a protocol for a single centre, open label, randomised controlled trial, using a two-arm design to determine whether exposure-guided dosing (therapeutic drug monitoring-TDM) of EC-MPS results in a higher proportion of participants achieving targets of MPA exposure compared with fixed drug dosing in participants with LN.

Key messages

- This study determines whether TDM-guided dosing of EC-MPS results in a higher proportion of participants in achieving established targets of MPA exposure compared with the standard fixed dosing in participants with biopsy-proven LN.

- This study also determines whether there is a difference in the complete and partial remission rates between participants on fixed drug dosing and exposure-controlled dosing of MPS in LN in induction group and renal relapse in the maintenance group.

- This study will try to validate a limited blood sampling protocol to measure exposure to MPA using a $0-12 \mathrm{~h}, 17$ sample-based area under curve.

Strengths and limitations of this study

- This prospective randomised study is designed specifically to test whether exposure controlled dosing results in a higher proportion of participants achieves target exposure levels of MPA compared with exposure controlled dosing.

- Size of the sample studied limits the power of observations. 
since 1995 in the USA and 1996 in Europe. ${ }^{1}$ A second MPA product, the enteric-coated mycophenolate sodium (EC-MPS) became available subsequently. Clinical trials have shown that EC-MPS is therapeutically equivalent to MMF with comparable safety profiles and equimolar doses of the two drugs producing equivalent MPA exposures. ${ }^{2} 3$ The favourable experience with MMF in transplant recipients resulted in it being used in trials involving participants with autoimmune and other immunologically mediated renal diseases. ${ }^{4-8}$ The Aspreva Lupus Management Study group (ALMS) found rates of adverse events, particularly infection, between MMF and intravenous cyclophosphamide to be similar in lupus nephritis (LN) ${ }^{9}$ Furthermore, a recent meta-analysis of randomised controlled trials showed that MMF was superior in inducing remission in severe LN and had fewer side effects compared with pulsed cyclophosphamide. ${ }^{10}$ Other clinical trials reported similar results with EC-MPS. ${ }^{11-14}$ The Therapeutic Goods Administration of Australia approved EC-MPS-Myfortic (http://www.novartis.com.au/DownloadFile.aspx?t=pf=myf. pdf) for the treatment of classes III, IV, V and V LN. ${ }^{15}$

\section{Dosing and therapeutic drug monitoring of MIMF/MPS}

MMF is usually administered at a fixed dose of $1000 \mathrm{mg}$ twice daily in transplant recipients (independent of weight) and modified according to clinical tolerance. The randomised controlled trial, the Adaption de Posologie du MMF en Greffe Renale (APOMYGRE) and the fixed dose versus concentration controlled (FDCC) studies investigated the benefit of using therapeutic drug monitoring (TDM) but results were not consistent. ${ }^{16} 17$ APOMYGRE showed a reduction in acute rejection whereas the FDCC study had a neutral effect. To date, there is currently no consensus on using TDM of MPA to optimise MMF use in patients with transplants. ${ }^{18-21}$

The dose of MMF for induction and maintenance therapy in $\mathrm{LN}$ varies from 2 to 3 gdaily. ${ }^{22}$ In the ALMS study, the median MMF dose was $42 \mathrm{mg} / \mathrm{kg}$ body weight by $20-24$ weeks and $91.3 \%$ of participants tolerated daily doses of $2.5-3 \mathrm{~g}$ daily. ${ }^{23}$ In a study by Pietruck $e t$ al, $720 \mathrm{mg}$ of MPS and $1000 \mathrm{mg}$ of MMF were found to deliver near equimolar exposures of MPA. ${ }^{24}$

\section{Interindividual variability of MPA}

Factors affecting the interpatient variability of MPA have been extensively investigated in transplant recipients and are likely to be similar in LN. ${ }^{25} 26$ The clearance of MPA depends on its non-protein bound fraction. ${ }^{26}$ Therefore, patients with reduced renal function and hypoalbuminaemia have higher free fractions of MPA, theoretically resulting in higher MPA clearance and a lower MPA exposure. Furthermore, cotherapy with calcineurin inhibitors, phosphate binders, steroids and rifampicin may affect MPA pharmacokinetics. ${ }^{26}$ Nonetheless, the MPA concentration-effect relationship is more likely to be linear in LN patients treated with MMF and steroids, as opposed to transplant recipients who are treated with many immunosuppressive agents. ${ }^{27}$

\section{TDM of MPA in LN}

Neumann $e t a t^{28}{ }^{29}$ reported on the value of measuring MPA plasma concentrations in patients with autoimmune diseases. In their study, there was a weak correlation between the $12 \mathrm{~h}$ trough (C0) MPA concentrations and the area under the curve (AUC), with remission being associated with higher MPA trough concentrations $(\geq 3.5 \mathrm{mg} / \mathrm{L})$. There was a clustering of adverse events in participants with a high MPA exposure, thus refining the therapeutic window. In contrast, studies in renal transplant participants showed tolerability correlated poorly with MPA concentrations. Neumann et al proposed in their exploratory study that MPA trough levels between 3.5 and $4.5 \mathrm{mg} / \mathrm{L}$ be used for maintenance of remission and prevention of adverse events. Roland $e t a l,{ }^{30}$ in a prospective pharmacokinetic study of MMF in patients treated for systemic lupus erythematosus, found a high interindividual variability of MPA AUC levels. The therapeutic range of MPA has to be better defined with the clinical response to exposure-controlled dosing in patients with LN.

\section{Limited sample strategy}

Measurement of AUC 0-12 h has been well documented in transplant literature and a target AUC 0-12 of 30$60 \mathrm{mg} / \mathrm{L} / \mathrm{h}$ is advised to reduce the risk of rejection. ${ }^{31} 32$ For treatment with MMF, LSS was suggested as an alternative because MPA AUC 0-12 was laborious and expensive. ${ }^{33}$ LSS has been trialled for EC-MPS showing that time points from the initial half of the full concentration time profile produced near identical results. ${ }^{35}$ Neuman et al found that MPA exposure expressed as MPA $\mathrm{AUC}_{0-12}$ was comparable in patients with autoimmune diseases and renal patients with transplants treated with EC-MPS. ${ }^{36}$ They suggested an LSS for estimating MPA exposure could be valid in autoimmune disorders. A recent study using MPS suggested an AUC over $8 \mathrm{~h}$ could be calculated with four blood samples, correlating favourably with an $\mathrm{AUC}_{0-12} \cdot{ }^{37}$

Our study will include participants who have blood sampled over a period of 8 or $12 \mathrm{~h}$. For the $8 \mathrm{~h}$ sampling period, the $\mathrm{AUC}_{0-12}$ will be calculated imputing the predose sample value for the $12 \mathrm{~h}$ sample value. With few studies in LN treated with MPS formulations measuring the relationship of MPA levels to efficacy and toxicity, this prospective randomised controlled trial will add more information on the pharmacokinetics and pharmacodynamics of MPA in LN. ${ }^{38} 39$

\section{AIM}

This study is to determine whether TDM-guided dosing of EC-MPS results in a higher proportion of participants achieving targets of MPA exposure in LN compared with fixed drug dosing. 


\section{METHODS/DESIGN}

\section{Study design and setting}

The study is a prospective, open-label, randomised controlled trial. Participant population: Participants will be selected from either inpatients at Royal Brisbane and Women's Hospital (RBWH) Renal and Rheumatology Departments or patients attending the Renal Rheumatology Lupus Vasculitis Clinic. This study will be carried out in collaboration with the Burns, Trauma and Critical Care Research Centre, School of Medicine, The University of Queensland and Department of Chemical Pathology, Pathology Queensland, Queensland Health.

The interventions will comprise of two groups:

\section{Intervention: control}

Group 1: Fixed dose regime-oral EC-MPS $30 \mathrm{mg} / \mathrm{kg}$ body weight to induce remission. MPS dosage will be reduced by $180 \mathrm{mg}$ twice daily on achieving complete remission or if there are side effects such as diarrhoea or leucopenia (total white cell count $<3500 / \mathrm{mm}^{3}$ or opportunistic infections).
Intervention: experimental

Group 2: Exposure controlled dose-oral EC-MPS dose will be titrated according to the $\mathrm{AUC}_{0-12}$ adjusted to a target $\mathrm{AUC}_{0-12}$ of $40-60 \mathrm{mg} / \mathrm{L} / \mathrm{h}$. The dosage will be reduced if the $\mathrm{AUC}_{0-12}$ is above $60 \mathrm{mg} / \mathrm{L} / \mathrm{h}$ and once there is complete remission, an AUC of $30-50 \mathrm{mg} / \mathrm{L} / \mathrm{h}$ will be maintained.

All participants in the control and intervention groups will undergo pharmacokinetic analysis of MPA.

Other than EC-MPS dosing, both groups will be given similar management of LN including treatment with corticosteroids. The illustration of the study design is presented in Figure 1.

\section{Identification of eligible participants}

Participants with biopsy-proven classes III/IV/V LN who fulfil the inclusion and exclusion criteria are eligible for the study. The inclusion criterion has been kept as broad as possible to represent the participant population and the exclusion criteria as restricted as possible to maximise validity and generalisability of findings.

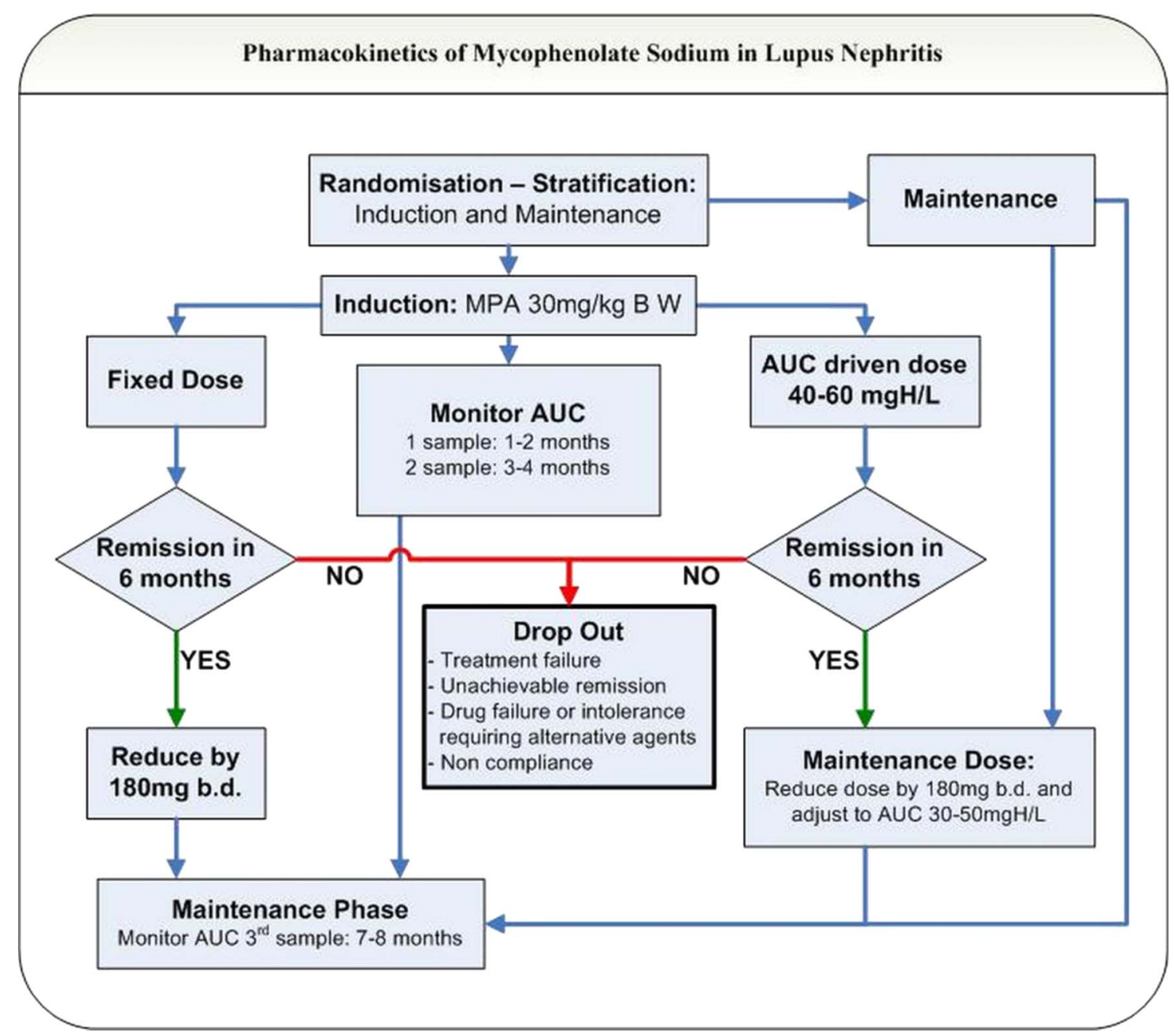

Figure 1 Pharmacokinetics of mycophenolate sodium in lupus nephritis (POEMSLUN) study design. 


\section{Inclusion criteria}

All participants 18 years of age and above with classes III/IV/V LN proven by renal biopsy and have been on EC-MPS for more than 2 weeks either as maintenance or induction therapy will be eligible for this study.

\section{Exclusion criteria}

Participants will be excluded if there is a history of psychological illness, a condition which interferes with their ability to understand or comply with the requirements of the study or who are unable to give consent. Pregnant, nursing (lactating) women and women planning on getting pregnant during the study period and not using contraception will be excluded. Finally, those with a history of malignancy treated or untreated within the past 5 years (with the exception of localised basal or squamous cell carcinomas of the skin) regardless of local recurrence or metastases will also be excluded from the study.

\section{Informed consent}

At the recommendation of the treating clinician who will seek permission from the participant or authorised surrogate to be approached by the investigator, the investigator/s will seek informed consent or assent from potential trial participants or authorised surrogate. This will occur through a verbal presentation of the written consent document, which the participant or authorised surrogate would then be given sufficient time to consider before consenting to the study or not.

\section{Participants}

Participants are randomised in permuted block sizes of 2 and 4 with the $33 \%$ and $66 \%$, respectively, and stratified for induction and maintenance therapy with EC-MPS. Masking of investigators and participants will not be possible. Nonetheless, the laboratory staff analysing the pharmacokinetics of MPS will be masked to the treatment allocation. An individual's participation in the study will cease at the end of the study period, which will be 12 months after the last participant is recruited. The recruitment period is 36 months. It is anticipated that the average follow-up time will be 24 months, assuming a steady recruitment rate of 12 participants per year.

The participants will have regular clinic appointments and follow-up to improve adherence. A trial pharmacist will be involved with the study and will detail the changes in medication doses and monitor participant adherence.

\section{Adverse events}

Adverse events are defined as any untoward medical event (clinical or laboratory) experienced by a participant during the course of this clinical trial and considered by the investigator to be related to the study. All adverse events will be recorded on the Adverse Event page of the case report form and reported immediately to the Human Research and Ethics Committee in writing.

Adverse events will be reported from the day of consent. The responsible investigator will determine whether the degree of any untoward event warrants removal of any participant from the study. They will institute appropriate diagnostic and therapeutic measures and keep the participant under observation for as long as is medically indicated.

\section{OUTCOME MEASURES \\ Primary}

This is a study to determine whether TDM-guided dosing of EC-MPS results in a higher proportion of participants achieving established targets of MPA exposure compared with the standard empirical dosing in participants with biopsy-proven LN.

\section{Secondary}

This study will also determine whether there is a difference in the complete and partial remission rates between participants on fixed drug dosing and exposure controlled dosing of MPS in LN in induction group and renal relapse in the maintenance group.

Complete remission is defined as a decrease in urinary protein measured over $24 \mathrm{~h}$ to less than $0.3 \mathrm{~g} /$ $24 \mathrm{~h}$, a urine protein/creatine ratio of less than $0.3 \mathrm{~g} / \mathrm{g}$ $(30 \mathrm{mg} / \mathrm{mmol})$ with a normal urinary sediment, normal serum albumin and stabilisation $( \pm 25 \%)$ or improvement in serum creatine levels at week 24 from the first sample. Partial remission is defined as stabilisation $( \pm 25 \%)$ or improved renal function (but still not to normal with reduction of proteinuria by more than $50 \%$ ranging between 0.3 and $3 \mathrm{~g} / 24 \mathrm{~h}$ and a serum albumin of more than $30 \mathrm{~g} / \mathrm{L} .{ }^{40}$ Renal relapse is defined as "recrudescence of renal disease after an initial response demonstrated by a recent increase in serum creatine by $>50 \%$ with active urinary sediment and or increase in proteinuria to $3.5 \mathrm{~g}$ /day or greater." ${ }^{41}$ Proteinuria will be measured using the urine protein to creatine ratio or by measuring the $24 \mathrm{~h}$ urinary protein excretion. The urine protein-to-creatine ratio is numerically equal to $24 \mathrm{~h}$ urinary protein excretion in grams.

Secondary outcome measures include the time required to achieve complete or partial remission, the assessment of improvement with the SLE Disease Activity Index (SLEDAI) score as well as C3, C4 and anti-double-stranded DNA (anti-dsDNA) levels, the development of a pharmacokinetic model that can be used to develop MPS dosing recommendations in LN participants treated with MPS, validating the LSS for determining $\mathrm{AUC}_{0-12}$, and the evaluation of the cost effectiveness of TDM analysis of MPS.

An individual who is masked to the study allocation and not involved in the clinical care of the participant will adjudicate outcome measures. 


\section{Data collection}

Clinical and demographic data are collected for each participant, including age, gender, weight, height, allergies, clinical information, other comorbidities, concomitantly prescribed drugs, serious adverse events, safety and treatment outcomes (clinical and/or immunological improvement) including SLEDAI scores (Table 1).

Laboratory investigations are performed consisting of renal function assessments, urine sediment examination, $24 \mathrm{~h}$ urinary protein and creatine measurement, estimated-glomerular filtration rate (modification of diet in renal disease-glomerular filtration rate $(\mathrm{mL} / \mathrm{min} /$ $\left.1.73 \mathrm{~m}^{2}\right)=175 \times(\mathrm{S}$. Cr $)-1.154 \times($ age $)-0.203 \times(0.742$ if female $) \times(1.212$ if African-American) (conventional units $)^{42}$ ), liver function tests, complement components C3 and C4, antinuclear antibody (ANA), anti-dsDNA and pharmacokinetic analysis of MPA.

The above information will be collected every 12 weeks and the presence of any serious adverse effects will be continually monitored and recorded during the study period. It is anticipated that the average follow-up time will be 18 months assuming a constant recruitment rate.

\section{Data analysis}

Blood samples will be collected by a dedicated research nurse at 15 time points for the $8 \mathrm{~h}$ group and 17 samples for the $12 \mathrm{~h}$ group, including the time points from previously described LSS data for MPS at 0, 1.5, 4 and $8 \mathrm{~h}$ postdose, on three different occasions. Blood samples for the $8 \mathrm{~h}$ group will be collected at half hourly intervals initially until the $7 \mathrm{~h}$ and the last sample at the eighth hour. Blood samples for the $12 \mathrm{~h}$ group will be collected as for the $8 \mathrm{~h}$ group along with further samples at the 10th and 12th hour. Samples will be kept on ice until centrifugation ( $3000 \mathrm{rpm}$ for $10 \mathrm{~min}$ ) and will then be analysed by high-performance liquid chromatography at Pathology Queensland (Royal Brisbane and Women's Hospital, Herston, Australia).

Induction phase: Blood sampling at three different time points; first sample at 1-2 months, second at 3-4 months and the third at 7-9 months.

Maintenance phase: Pharmacokinetic study will be performed only once at the time of entry. The pharmacokinetic values will be calculated using non-compartmental methods. The $\mathrm{AUC}_{0-12}$ will be calculated using the trapezoidal rule; $\mathrm{CL}=$ Dose/AUC; $\mathrm{Cmax}$ will be the observed value; apparent terminal elimination rate constant $(\lambda z)$ will be determined from log-linear least squares regression analysis of concentrations from 4 to $8 \mathrm{~h}$. $\mathrm{Vz}=\mathrm{CL} / \lambda \mathrm{z}$; apparent elimination half-life $=\ln ^{2} / \lambda z$.

\section{Statistical methods}

A sample size of 32 participants is projected with 16 in each group. The sample required to test the ability for TDM-guided dosing to achieve target MPS exposure compared with no TDM, is based on pharmacokinetic data from patients with $\mathrm{LN}$ by Lertdumrongluk et $a l^{43}$

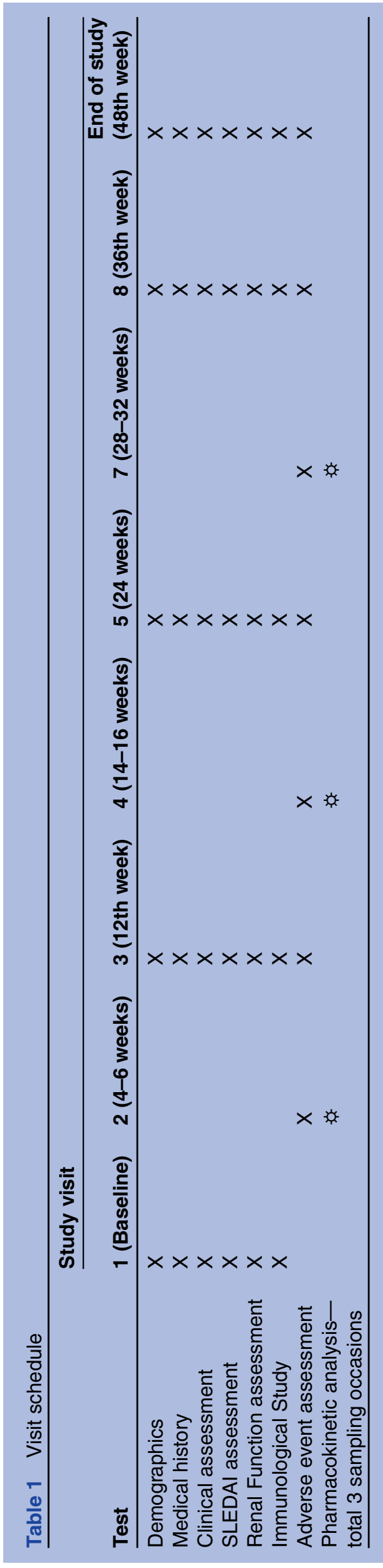


Using a 95\% CI, predicting that $50 \%$ of patients will have exposures under the target range requires a total sample of 30 participants. We will collect data from 32 participants assuming that there was less than a $10 \%$ attrition rate. ${ }^{44}$

Comparisons between groups for the outcome variable for complete and partial remission will be performed using $\chi^{2}$ or Fisher's exact tests followed by a multivariable logistic regression analysis to estimate the association between outcome and the groups. The secondary outcome of interest, namely, time to complete/partial remission will be evaluated using Kaplan-Meier survival curves, log-rank test. Multivariable Cox regression models will be used to estimate the effect of group adjusting for other covariates. Student $t$ test or Mann-Whitney U test are used to compare the continuous variables. Differences in infection rates (number of infections/total follow-up time) and serious adverse events between the two groups will be analysed by $\mathrm{Z}$ tests. All data will be analysed on an intention-to-treat basis, and a significance level of 0.05 will be assumed.

\section{Ethical considerations and dissemination}

The Human Research and Ethics Committee of the RBWH have approved this study. The investigators will comply with and conduct the study in accordance with Good Clinical Practice Guidelines, the principles that have their origins in the 'Declaration of Helsinki' adopted by the World Medical Association in October 1996, the National Health and Medical Research Council (NHMRC) National Statement on Ethical Conduct in Human Research (2007) or replacement, and or other relevant NHMRC publication or guideline that relate or may relate to clinical trials. ${ }^{45}$

Withdrawal of study medication may be made at the discretion of the treating physician or investigator following an adverse event. However, participants will continue to be followed for the duration of the study if possible, even if they have stopped treatment. This will include attendance at scheduled trial visits, and data collection, particularly of outcomes. The investigator may withdraw a participant from study medication at any time if it is felt to be in the best interests of the participant, where there is disease progression or non-adherence to study medication. Under such circumstances, participants will continue to be monitored for outcome events and will attend scheduled trial visits so that results can be analysed on an intention to treat basis. If a participant withdraws consent for the study they shall be withdrawn from the study. Any participant is free to withdraw their consent at any time without the need to justify their decision.

We plan to publish the results in medical journals and present the de-identified information at conferences.

\section{Protection of participant confidentiality}

Participants' records and the data generated by the study will be confidential in line with the recommendations of the Australian NHMRC and locally applicable laws. Standardised case report forms will be provided for each participant on this study. The participants in this study will be identified only by initials and subject number on these forms. Any information that may identify a participant will be excluded from data presented in the public arena. Data will only be shared among the investigators. Individual participant's medical information may be given to the treating clinician where deemed clinically necessary. De-identified information will not be released. Data collected as part of this trial will be stored on a computer data base held by the principal investigator in a secure, lockable location. The data will be stored for 15 years after completion of the project in accordance with the revision of the Joint NHMRC/Australian Vice Chancellors' Committee (AVCC) statement and guidelines on research practice. ${ }^{45}$

\section{DISCUSSION}

Several studies have demonstrated that MMF/MPS is effective at gaining control of severe LN. The variations in pharmacokinetics of MPA/MPS and the factors causing these differences have been studied in transplant recipients but are not well documented in those with LN. The primary objective of this study is to examine whether exposure controlled MPS dosing results in higher proportion of participants achieving targets of MPA exposure compared with standard empiric dosing in participants with LN. Secondary objectives include the efficacy of exposure-controlled MPS dosing in LN with TDM, the relationship between the disease activity of LN and MPA blood concentrations (pharmacodynamics), and using the data to develop dosing recommendations for MPS in LN. Defining the optimal therapeutic range for MPA is likely to help improve outcomes in participants with $\mathrm{LN}$ treated with MPS. The consequences of failure of immunosuppression in LN can be severe and can lead to end-stage renal failure. By personalising MPS dosing for participants with LN using TDM, it may be possible to maximise therapeutic efficacy while preventing significant complications, therapeutic failure and toxicity.

\section{CURRENT STATUS OF THE STUDY}

The study has started and is in its early stages. The recruitment rate will be assessed periodically and if the target is unlikely to be met, we will invite other nephrology centres in Queensland to participate in the study.

\section{Author affiliations}

${ }^{1}$ Department of Renal Medicine, Royal Brisbane and Women's Hospital, Brisbane, Queensland, Australia

${ }^{2}$ School of Medicine, The University of Queensland, Brisbane, Queensland, Australia

${ }^{3}$ Department of Intensive Care Medicine, Royal Brisbane and Women's Hospital, Brisbane, Queensland, Australia

${ }^{4}$ Department of Clinical Pharmacology and Rheumatology, Royal Brisbane and Women's Hospital, Brisbane, Queensland, Australia 
${ }^{5}$ Department of Chemical Pathology, Pathology Queensland, Brisbane, Queensland, Australia

${ }^{6}$ Department of Pharmacy and Intensive Care Medicine, Royal Brisbane and Women's Hospital, Brisbane, Queensland, Australia

Acknowledgements The authors would like to thank Dr Sree Krishna Venthurupalli and Dr Jennifer Martin, members of Data Safety monitoring board and Dr Shankar Viswanathan for advice on statistical methods.

Contributors DR, GTJ and JAR designed the study and wrote the protocol. $\mathrm{HH}, \mathrm{JL}$ and RGF adviced and reviewed the study protocol and ethics application. PK provided input and will be involved with the recruitment component of the study. JAR, JU and BCMcW will be involved in the analysis of samples. AL, MLP, MJR and RR will be involved in the collection of blood samples and data collection. AL assisted in preparing the manuscript. All authors read and approved the final version of the manuscript.

Funding This study is funded by a Research grant from the RBWH Research Foundation and an unrestricted grant Novartis Australia. The study funders have no role in study design; collection, management, analysis and interpretation of data; writing of the report; and the decision to submit the report for publication. The investigators will have ultimate authority over each of these activities.

Competing interests DR and GTJ were principal investigators for Ascertain study sponsored by Novartis Australia (pte) limited and Novartis India (pte) limited, respectively.

Ethics approval Human Research \& Ethics Committee, Royal Brisbane \& Women's Hospital, Metronorth Health Services District.

Provenance and peer review Not commissioned; internally peer reviewed.

Data sharing statement Data sets are available with the corresponding author dwarakanathan_ranganathan@health.gld.gov.au.

Open Access This is an Open Access article distributed in accordance with the Creative Commons Attribution Non Commercial (CC BY-NC 3.0) license, which permits others to distribute, remix, adapt, build upon this work noncommercially, and license their derivative works on different terms, provided the original work is properly cited and the use is non-commercial. See: http:// creativecommons.org/licenses/by-nc/3.0/

\section{REFERENCES}

1. Meier-Kriesche HU, Li S, Gruessner RW, et al. Immunosuppression evolution in practice and trends, 1994-2004. Am J Transplant 2006:6:1111-31.

2. Salvadori M, Holzer $\mathrm{H}$, de Mattos $\mathrm{A}$, et al. Enteric-coated mycophenolate sodium is therapeutically equivalent to mycophenolate mofetil in de novo renal transplant participants. Am J Transplant 2004;4:231-6.

3. Budde K, Bauer S, Hambach $\mathrm{P}$, et al. Pharmacokinetic and pharmacodynamic comparison of enteric-coated mycophenolate sodium and mycophenolate mofetil in maintenance renal transplant participants. Am J Transplant 2007;7:888-98.

4. Stassen PM, Kallenberg CG, Stegeman CA. Use of mycophenolic acid in non-transplant renal diseases. Nephrol Dial Transplant 2007;22:1013-19.

5. Appel GB, Radhakrishnan J, Ginzler EM. Use of mycophenolate mofetil in autoimmune and renal diseases. Transplantation 2005;80 (2 Suppl):S265-71

6. Chan TM, Li FK, Tang CS, et al. Efficay of mycophenolate moeftil in participants with diffuse proliferative Lupus Nephritis. N Engl J Med 2000;343:1156-62.

7. Chan TM, Tse KC, Tang CS, et al. Long-term study of mycophenolate moeftil as continuos induction and maintenance treatment for diffuse proliferative lupus nephritis. J Am Soc Nephrol 2005;16:1076-84

8. Laccarino L, Rampuddda M, Canova M, et al. Mycophenolate mofetil: what is its place in the treatment of autoimmune rheumatic disease? Autoimmu Rev 2007;6:190-5.

9. Sinclair A, Appel G, Dooley MA, et al. Mycophenolate mofetil as induction and maintenance therapy for lupus nephritis: rationale and protocol for the randomized, controlled Aspreva Lupus Management Study (ALMS). Lupus 2007;16:972-80.
10. Zhu B, Chen N, Lin Y, et al. Mycophenolate mofetil in induction and maintenance therapy of severe lupus nephritis: a meta-analysis of randomized controlled trials. Nephrol Dial Transplant 2007;22:1933-42.

11. Kitiyakara C, Ophascharoensuk V, Chanrisokulchai, et al. Treatment of lupus nephritis and primary glomerulonephritis with enteric coated mycophenolate sodium. Clin Nephrol 2008;69.2:90-101.

12. Mak S, Lo KY, Lo MW, et al. Efficacy of enteric-coated mycophenolate sodium in participants with active lupus nephritis. Nephrology 2007;13.4:331-6.

13. Traitanon $\mathrm{O}$, Avihingsanon $\mathrm{Y}$, Kittikovit $\mathrm{V}$, et al. Efficacy of enteric-coated mycophenolate sodium in participants with active lupus nephritis. Lupus 2008;17.8:744-51.

14. Willeke $\mathrm{P}$, Schluter $\mathrm{B}$, Becker $\mathrm{H}$, et al. Mycophenolate sodium treatment in participants with primary Sjogren syndrome: a pilot trial 2007. Arthritis Res Ther2007:9:R115.

15. Weening JJ, D'Agati VD, Schwartz MM et al. Classification of glomerulonephritis in systemic lupus erythematosus revisited. Kidney Int 2004;65:521-30.

16. Le Meur $Y$, Büchler M, Thierry $A$, et al. Individualized mycophenolate mofetil dosing based on drug exposure significantly improves participant outcomes after renal transplantation. $A m \mathrm{~J}$ Transplant 2007;7:2496-503.

17. Van Gelder T, Silva HT, de Fijter JW, et al. Comparing mycophenolate mofetil drug monitoring regimens for de novo renal transplant participants: the fixed-dose concentration-controlled trial. Transplantation 2008;86:1043-51.

18. Gaston RS, Kaplan B, Shah T, et al. Fixed or controlled-dose mycophenolate mofetil with standard-or reduced dose calcineurin inhibitors: the Opticept Trial. Am J Transplant 2009;9:1607-19.

19. West-Thielke $P$, Kaplan B. Therapeutic monitoring of mycophenolic acid: is there clinical utility? Am J Transplant 2007;7:2441-2.

20. Knight SR, Morris PJ. Does the evidence support the use of mycophenolate mofetil therapeutic drug monitoring in clinical practice? A systematic review. Transplantation 2008;85:1675-85.

21. van Gelder T. Mycophenolate blood level monitoring : recent progress. Am J Transplant 2009;9:1495-9.

22. Ginzler EM, Dooley MA, Aranow C, et al. Mycophenolate mofetil or intravenous cyclophosphamide for lupus nephritis. $N$ Engl J Med 2005;353:2219-28.

23. Isenberg D, Appel G, Contrears, et al. Influence of race/ethinicity on response to lupus nephritis treatment: the ALMS study. Rheumatology 2010;49:128-40.

24. Pietruck F, Abbud-Fiho $M$, Vathsala $A$, et al. Conversion from mycophenolate mofetil to enteric coated mycophenolate sodium in stable maintenance transplant participants; pooled results from three international multicenter studies. Transplant Proc 2007;39:103-8.

25. De Winter BCM, Mathôt RAA, van Hest RM, et al. Therapeutic drug monitoring of mycophenolic acid: does it improve participant outcome? Expert Opin Drug Metab Toxicol 2007;3:251-61.

26. Bullingham RES, Nocholls AJ, Kamm BR. Clinical pharmacokinetics of mycophenolate mofetil. Clin Pharmacokinet 1998;34:429-55.

27. Hasseling DA, Van Gelder T. Genetic and non-genetic determinants of between-participant variablity in the pharmacokinetics of mycophenolic acid. Clin Pharmacol Ther 2005;78:317-21.

28. Neumann I, Haidinger $M$, Jäger $H$, et al. Pharmacokinetics of mycophenolate mofetil in participants with autoimmune diseases compared to renal transplant recipients. J Am Soc Nephrol 2003;14:721-7.

29. Neumann I, Fuhrmann H, Fang IF, et al. Association between mycophenolic acid 12-hour trough levels and clinical endpoints in participants with autoimmune disease on mycophenolate mofetil. Nephrol Dial Transplant 2008;23:3514-20.

30. Roland M, Barbet C, Paintaud G, et al. Mycophenolate mofetil in participants with systemic lupus erythematosus: a prospective pharmacokinetic study. Lupus 2009;8:441-7.

31. van Gelder T, Le Meur Y, Shaw LM, et al. Therapeutic drug monitoring of mycophenolate mofetil in transplantation. Ther Drug Monit 2006;28:145-54.

32. Shaw LM, Kaplan B, De Nofrio D, et al. Pharmacokinetics and concentration-control investigations of mycophenolic acid in adults after transplantation. Ther Drug Monit 2000;22:14-19.

33. Mathew BS, Fleming DH, Annapandian VM, et al. A reliable limited sampling strategy for the estimation of mycophenolic acid area under the concentration time curve in adult renal transplant participants in the stable post transplant period. Ther Drug Monit 2010;32:136-40.

34. Pawinski T, Hale M, Korecka M, et al. Limited sampling strategy for the estimation of mycophenolic acid area under the curve in adult renal transplant participants treated with concomitant tacrolimus. Clin Chem 2002;48:1497-504 
35. Sommerer C, Müller-Krebs S, Schaier M, et al. Pharmacokinetic and pharmacodynamic analysis of enteric-coated mycophenolate sodium: limited sampling strategies in renal transplant participants. Br J Clin Pharmacol 2010:69:346-57.

36. Neumann I, Fuhrmann H, Fang IF, et al. Pharmacokinetics of enteric-coated mycophenolate sodium: comparative study in participants with autoimmune disease and renal allograft. Expert Opin Pharmacother 2008;9:879-86.

37. Sánchez Fructuoso Al, Perez-Flores I, Calvo N, et al. Limited-sampling strategy for mycophenolic acid in renal transplant recipients' receiving enteric-coated mycophenolate sodium and tacrolimus. Ther Drug Monit 2012;34:298-305.

38. Hawker G, Gabriel S, Bombardier C, et al. A reliability study of SLEDAl: a disease activity index for systemic lupus erythematosus. $J$ Rheumatol 1998;20:657-60.

39. Mukhtyar C, Lee R, Brown D, et al. Modification and validation of the Birmingham Vasculitis Activity score (Version 3). Ann Rheum Dis 2009;68:1827-32.
40. Kidney Disease: Improving Global Outcomes (KDIGO) Glomerulonephritis Work Group. KDIGO Clinical Practice Guideline for Glomerulonephritis. Kidney Int. 2012;2:139-274.

41. Hill GS, Michel Delahousse M, Nochy D, et al. Outcome of relapse in lupus nephritis: roles of reversal of renal fibrosis and response of inflammation to therapy. Kidney Int. 2002;61:2176-86.

42. Levey AS, Coresh J, Greene T, et al. Chronic Kidney Disease Epidemiology Collaboration. Using standardized serum creatinine values in the modification of diet in renal disease study equation for estimating glomerular filtration rate. Ann Intern Med 2006;145:247-54.

43. Lertdumrongluk $\mathrm{P}$, Somparn $\mathrm{P}$, Kittanamongkolchai $\mathrm{W}$, et al. Pharmacokinetics of mycophenolic acid in severe lupus nephritis. Kidney Int 2010;78:389-95.

44. http://www.nss.gov.au/nss/home.nsf/pages/Sample+size+calculator

45. National Health and Medical Research Council; Australian Research Council and the Universities Australia. Australian code for the conduct of research 2007. http://www.nhmrc.gov.au/_files_nhmrc/ publications/attachments/e72.pdf. 